\title{
Points de petite hauteur sur une sous-variété d'un tore
}

\author{
Francesco Amoroso et Sinnou David
}

\begin{abstract}
We prove an almost optimal lower bound for the so called last 'geometric' minimum for the height of a subvariety $V$ of a power of the multiplicative group $\mathbb{G}_{m}^{n}$. More precisely, one shows that if $\boldsymbol{x}$ is a point of $V(\overline{\mathbb{Q}})$ which does not belong to a translate of a sub-torus of $\mathbb{G}_{m}^{n}$ lying in $V$, of height less than a function essentially linear in the inverse of the degree of $V$, then $\boldsymbol{x}$ belongs to a finite 'exceptional' subset of $V$. Thus, one proves 'up to an $\varepsilon$ ' the sharpest conjectures that can be formulated on this problem. Previously, the best known result in this direction was due to the second author and Philippon, who provided lower bounds which were inverse monomial in the degree of $V$ (Minorations des hauteurs normalisées des sous-variétés des tores, Ann. Scuola Norm. Sup. Pisa Cl. Sci. (4) 28 (1999), 489-543; Errata 29 (2000)).
\end{abstract}

\section{RÉSUMÉ}

Nous obtenons une minoration quasi optimale pour le dernier des minima dits « géométriques » d'une sous-variété $V$ d'une puissance du groupe multiplicatif $\mathbb{G}_{m}^{n}$. Plus précisément, nous montrons que si $\boldsymbol{x}$ est un point de $V(\overline{\mathbb{Q}})$ n'appartenant pas à un translaté d'un sous-tore de $\mathbb{G}_{m}^{n}$ contenu dans $V$, de hauteur majorée par une fonction essentiellement linéaire en l'inverse du degré de $V$, alors $\boldsymbol{x}$ appartient à un ensemble « exceptionnel » de cardinal fini. Ainsi, à un « $\varepsilon$ près », nous montrons les conjectures les plus précises que l'on peut formuler pour ce problème. Les meilleurs résultats connus précédemment, dus au second auteur et Philippon, fournissaient des minorations monomiales inverses en le degré de $V$ (Minorations des hauteurs normalisées des sous-variétés des tores, Ann. Scuola Norm. Sup. Pisa Cl. Sci. (4) 28 (1999), 489-543 ; Errata 29 (2000)).

\section{Introduction}

Dans un article récent (confer [AD03a]), nous avons montré une minoration presque optimale pour le « minimum essentiel » de la hauteur des points algébriques d'une sous-variété algébrique $V$ de $\mathbb{G}_{m}^{n}$ définie sur $\overline{\mathbb{Q}}$ et géométriquement irréductible. Nous nous proposons ici de compléter ce travail en montrant qu'une minoration de même qualité est en fait valable pour le dernier des minima dits « géométriques » pour la hauteur des points de $V$. Commençons par rappeler plus précisément ces différentes notions.

Soit $n$ un entier $\geqslant 1$. Dans ce travail, nous nous restreindrons pour simplifier à la compactification naturelle $\mathbb{G}_{m}^{n} \hookrightarrow \mathbb{P}^{n}$ du tore. ${ }^{1}$ Soit $V$ une sous-variété algébrique de $\mathbb{G}_{m}^{n}$, définie sur $\overline{\mathbb{Q}}$, et géométriquement irréductible ; le degré de $V$ sera celui de son adhérence de Zariski dans $\mathbb{P}^{n}$.

Received 22 March 2005, accepted in final form 17 October 2005.

2000 Mathematics Subject Classification 11G10, 11J81, 14G40.

Keywords: heights, Bogomolov's conjecture, small points.

This journal is (C) Foundation Compositio Mathematica 2006.

${ }^{1}$ L'essentiel des problématiques et une partie des résultats peuvent se formuler plus généralement pour une compactification équivariante du tore. Nous ne rentrerons pas dans ces considérations au cours de ce texte. 


\section{F. Amoroso et S. David}

De même, si $\boldsymbol{\alpha}=\left(\alpha_{1}, \ldots, \alpha_{n}\right)$ est un point de $\mathbb{G}_{m}^{n}(\overline{\mathbb{Q}})$, sa hauteur normalisée $\hat{h}(\boldsymbol{\alpha})$ sera la hauteur de Weil logarithmique et absolue du point $\left(1: \alpha_{1}: \cdots: \alpha_{n}\right) \in \mathbb{P}^{n}(\overline{\mathbb{Q}})$. Soit $\theta$ un nombre réel strictement positif. On note

$$
V(\theta):=\{\boldsymbol{\alpha} \in V(\overline{\mathbb{Q}}) \text { tel que } \hat{h}(\boldsymbol{\alpha}) \leqslant \theta\} .
$$

Le minimum essentiel de $V$ est alors :

$$
\hat{\mu}^{\mathrm{ess}}(V):=\inf \{\theta>0, \overline{V(\theta)}=V\} .
$$

Rappelons que le minimum essentiel de $V$ est nul si et seulement si $V$ est un translaté d'un sous-tore par un point de torsion : c'est un théorème de Zhang, confer [Zha92] pour les courbes et [Zha95] plus généralement (il s'agit de la variante torique du problème dit de Bogomolov).

Rappelons enfin la notion d'indice d'obstruction ; si $V$ est une sous-variété de $\mathbb{G}_{m}^{n}$, son indice d'obstruction, noté $\omega(V)$, est l'infimum des degrés des hypersurfaces de $\mathbb{G}_{m}^{n}$ contenant $V$. Nous pouvons maintenant rappeler le résultat principal de [AD03a, théorème 1.4] :

ThÉorème 1.1. Soit $V$ une sous-variété ${ }^{2}$ de $\mathbb{G}_{m}^{n}$, définie sur $\overline{\mathbb{Q}}$ et géométriquement irréductible. Alors, si $V$ n'est pas contenue dans un translaté d'un sous-tore strict $B \subsetneq \mathbb{G}_{m}^{n}$, on a, en notant $k$ la codimension de $V$ :

$$
\hat{\mu}^{\text {ess }}(V) \geqslant c(n) \omega(V)^{-1}(\log (3 \omega(V)))^{-\lambda(k)},
$$

où $c(n)$ est un nombre réel strictement positif, effectivement calculable en fonction de la dimension ambiante $n$, et $\lambda(k):=\left(9(3 k)^{(k+1)}\right)^{k}$.

Ce théorème est optimal à des facteurs logarithmiques près : en effet, la conjecture la plus fine que l'on peut faire sur le comportement de $\hat{\mu}^{\text {ess }}(V)$ en fonction de l'indice d'obstruction est la suivante [AD03a, conjecture 1.2] :

Conjecture 1.2. Soit $V$ une sous-variété de $\mathbb{G}_{m}^{n}$ qui n'est pas un translaté d'un sous-tore strict de $\mathbb{G}_{m}^{n}$. Notons $B$ le plus petit translaté d'un sous-tore de $\mathbb{G}_{m}^{n}$ contenant $V$, alors :

$$
\hat{\mu}^{\text {ess }}(V) \geqslant \frac{c(n)}{\omega(V, B)} .
$$

Ici $\omega(V, W)$ est l'indice d'obstruction de $V$ relatif à la sous-variété $W \supsetneq V$, introduit dans la définition 1.1 de $[\mathrm{AD} 03 \mathrm{a}]$, i.e. la quantité

$$
\min \left(\frac{\operatorname{deg}(Z)}{\operatorname{deg}(W)}\right)^{1 / \operatorname{codim}_{W}(Z)}
$$

où $Z$ parcourt l'ensemble des sous-variétés de $\mathbb{G}_{m}^{n}$ tels que $V \subseteq Z \subsetneq W$. On a donc :

$$
\omega(V, W) \leqslant \operatorname{deg}(V)^{1 / \operatorname{codim}_{W}(V)} .
$$

De plus, il est facile de voir, en tenant compte d'un résultat de Chardin sur la fonction de Hilbert géométrique (confer [Cha88, corollaire 2, chapitre 1, p. 8 et exemple 1, p. 9]), que :

$$
\omega\left(V, \mathbb{G}_{m}^{n}\right) \leqslant \omega(V) \leqslant n \omega\left(V, \mathbb{G}_{m}^{n}\right) .
$$

L'indice d'obstruction relatif sert donc à tenir compte du degré du groupe ambiant, pour obtenir des résultats (ou conjectures) plus fins.

Le meilleur résultat dans la direction de la conjecture 1.2 connu précédemment était dû au second auteur de ces notes et à Philippon (confer [DP99, théorème 1.2]) :

$$
\hat{\mu}^{\text {ess }}(V) \geqslant 2^{-41} \operatorname{deg}(V)^{-2} \log (\operatorname{deg}(V)+2)^{-2} .
$$

\footnotetext{
${ }^{2}$ D'une manière générale, toutes les sous-variétés de $\mathbb{G}_{m}^{n}$ considérées dans cet article seront supposées algébriques,
} définies sur $\overline{\mathbb{Q}}$ et géométriquement irréductibles. 


\section{Points DE PETITE hauteur}

Enfin, les premiers résultats quantitatifs concernant le minimum essentiel (minoration et non pas seulement non-nullité) sont dus d'une part à Bombieri et Zannier (confer [BZ95]) et W. Schmidt (confer [Sch96]) d'autre part qui ont obtenu des minorations pluriexponentielles en le degré de $V$. Le lecteur pourra se reporter à [Amo04] et à [Dav] pour plus de détails sur ces questions. Il convient de noter que toutes ces minorations ne dépendent pas d'un corps de définition de $V$, et ne sont fonctions que d'invariants géométriques.

Dans ce texte, nous nous intéresserons à des quantités plus fines que le minimum essentiel. Dans [DP99], les auteurs ont introduit une série de « minima géométriques » notée $\hat{\mu}_{j}^{\circ}(V)$ dont nous rappelons ici la définition. Notons $\operatorname{pour}^{3} j=1, \ldots, \operatorname{dim}(V)$

$$
\hat{\mu}_{j}^{\circ}(V)=\sup _{Y} \inf \{\hat{h}(\boldsymbol{\alpha}), \boldsymbol{\alpha} \in(V \backslash Y)(\overline{\mathbb{Q}})\}
$$

où le supremum est pris sur les fermés de Zariski $Y$ de $V$ de la forme $Y=Y_{1} \cup Y_{2}$, où $Y_{1}$ est une réunion finie de sous-variétés de $V$ de codimension $j$ dans $V$ et $Y_{2}$ est une réunion finie ${ }^{4}$ de translatés de sous-tores de $\mathbb{G}_{m}^{n}$ contenus dans $V$ et de codimension $<j$ dans $V$.

On notera que cette suite diffère de la suite usuelle des minima successifs pour la hauteur (comme introduite par Zhang dans [Zha95]) :

$$
\hat{\mu}_{j}(V)=\sup _{\substack{Y \subset V, \operatorname{codim}_{V}(Y)=j}} \inf \{\hat{h}(\boldsymbol{\alpha}), \boldsymbol{\alpha} \in(V \backslash Y)(\overline{\mathbb{Q}})\}
$$

par le fait que l'on exclut aussi les translatés de sous-tores au lieu de se placer simplement en codimension $j$.

Par définition (on notera que la première égalité n'est valable que lorsque $V$ n'est pas un translaté d'un sous-tore),

$$
\hat{\mu}^{\text {ess }}(V)=\hat{\mu}_{1}^{\circ}(V) \geqslant \hat{\mu}_{2}^{\circ}(V) \geqslant \cdots \geqslant \hat{\mu}_{\operatorname{dim}(V)}^{\circ}(V) .
$$

La suite des minima géométriques est liée aux minima de la hauteur sur l'ouvert $V^{\circ}$, dont nous rappelons la définition et les principales propriétés. Suivant Bombieri, Zannier et Schmidt, pour tout sous-tore $H$ non trivial de $\mathbb{G}_{m}^{n}$, notons $V^{a}(H)$ la réunion des translatés de $H$ contenus dans $V$. Notons également $V^{a}$ la réunion des $V^{a}(H)$, pour $H$ parcourant l'ensemble des sous-tores de $\mathbb{G}_{m}^{n}$ de dimension $\geqslant 1$, et $V^{\circ}$ son complémentaire

$$
V^{\circ}:=V \backslash V^{a}
$$

L'ensemble $V^{a}$ est un fermé de Zariski ; de plus, il existe un ensemble fini $\left\{H_{1}, \ldots, H_{m}\right\}$ de sous-tores de dimension $\geqslant 1$ tels que

$$
V^{a}=\bigcup_{i=1}^{m} V^{a}\left(H_{i}\right)
$$

(confer [Sch96, Theorem 1]) et, pour chaque sous-tore $H$ l'ensemble $V^{a}(H)$ est un fermé de Zariski (confer [Sch96, Theorem 2]). Par contre, $V^{a}(H)$ n'est pas en général une réunion d'un nombre fini de translatés de sous-tores de $\mathbb{G}_{m}^{n}$ (confer le deuxième exemple ci-dessous).

Notons que l'ouvert $V^{\circ}$ peut être vide : il suffit pour cela que le stabilisateur $\operatorname{Stab}(V)$ de $V$ soit de dimension $\geqslant 1$. Inversement, $\operatorname{si} \operatorname{Stab}(V)$ est discret, $V^{\circ}$ est non vide.

Ainsi, les $\hat{\mu}_{j}^{\circ}(V)$ ne sont en fait pas tout à fait les minima successifs de la hauteur sur l'ouvert $V^{\circ}$ (et ce, même si $\operatorname{Stab}(V)$ est discret : il suffit par exemple que l'un des fermés $V^{a}(H)$ que l'on

\footnotetext{
${ }^{3}$ Par convention l'infimum sur l'ensemble vide vaut $+\infty$.

${ }^{4}$ En fait, la définition des $\hat{\mu}_{j}^{\circ}(V)$ de [DP99] est légèrement ambiguë. On pourrait la comprendre soit en prenant naturellement $Y_{2}$ comme ici, soit en prenant pour $Y_{2}$ la réunion des $V^{a}(H)$ où la codimension de $H$ est $<j$. Les résultats démontrés dans loc. cit. utilisent toutefois la même définition qu'ici et permettent de lever l'ambiguïté.
} 


\section{F. Amoroso et S. David}

retire soit de dimension $>\operatorname{dim}(H)$ ). Notons de plus que le minimum absolu de la hauteur sur $V^{\circ}$ peut être nul : il suffit que $V^{\circ}$ contienne un point de torsion. Toutefois, $\hat{\mu}_{\operatorname{dim}(V)}^{\circ}(V)$ n'est pas nul et peut même être minoré en fonction de $n$ et du degré de $V$ (cela résulte des travaux de Bombieri et Zannier et de Schmidt ; confer [BZ95, Sch96]).

Illustrons les notions précédentes à l'aide de quelques exemples :

Exemples. Si $V=\{\alpha\} \times \mathbb{G}_{m}^{n-1}$, où $\alpha \in \overline{\mathbb{Q}}^{*}$, alors $\hat{\mu}^{\text {ess }}(V)=\hat{h}(\alpha)$. Par contre, $V^{\circ}=\emptyset$, et $\hat{\mu}_{1}^{\circ}(V)=\cdots=\hat{\mu}_{n-1}^{\circ}(V)=+\infty$.

Si maintenant $\mathcal{C}$ est une courbe contenue dans $\mathbb{G}_{m}^{2}$, de stabilisateur discret, et si $V=\mathcal{C} \times \mathbb{G}_{m} \subset \mathbb{G}_{m}^{3}$, alors, on a encore $\hat{\mu}^{\text {ess }}(V)=\hat{\mu}^{\text {ess }}(\mathcal{C})$ et $V^{\circ}=\emptyset$. Par contre,

$$
\hat{\mu}_{1}^{\circ}(V)=\hat{\mu}^{\text {ess }}(V)=\hat{\mu}^{\text {ess }}(\mathcal{C}),
$$

puisque $V$ n'est pas un translaté d'un sous-tore mais $\hat{\mu}_{2}^{\circ}(V)=\hat{\mu}^{\text {ess }}(\mathcal{C})$ bien que $V$ soit une réunion de translatés de sous-groupes de codimension 1.

Ces considérations montrent que non seulement les $\hat{\mu}_{j}^{\circ}(V)$ reflètent assez bien le comportement de la hauteur sur l'ouvert $V^{\circ}$, mais ils prennent également en compte la variation de la hauteur sur $V$ tout entier, notamment lorsque son stabilisateur n'est pas discret.

Nous nous proposons d'étudier le dernier de ces minima géométriques, $\hat{\mu}_{\operatorname{dim}(V)}^{\circ}(V)$ que nous noterons pour alléger $\hat{\mu}^{\circ}(V)$ dans la suite.

On peut aussi interpréter la quantité $\hat{\mu}^{\circ}(V)$ comme un infimum des minima essentiels des sousvariétés de $V$; plus précisément, nous verrons au lemme 2.2,

$$
\hat{\mu}^{\circ}(V)=\inf _{Z} \hat{\mu}^{\text {ess }}(Z)
$$

où l'infimum est pris sur les sous-variétés $Z$ de $V$ qui ne sont pas contenues dans un translaté $B$ d'un sous-tore de $\mathbb{G}_{m}^{n}$ tel que $B \subseteq V$.

Avant d'énoncer des conjectures précises sur le comportement de $\hat{\mu}^{\circ}(V)$, introduisons encore une notion : on appelle indice d'interpolation de $V$, noté $\tilde{\delta}(V)$, le plus petit entier $\delta$ pour lequel l'idéal de définition $\Im(V)$ est engendré en degré $\leqslant \delta$. Un indice d'interpolation relatif à une variété $W$ intermédiaire est plus généralement introduit dans la définition 1.8 de [AD03a] : si $V \subsetneq W$, le plus petit entier $\delta$ tel qu'il existe des sous-variétés $Y_{1}, \ldots, Y_{t}$ de $W$ de codimension 1 dans $W$ et degré $\leqslant \delta$ telles que $V=Y_{1} \cap \cdots \cap Y_{t}$ est l'indice d'interpolation relatif $\tilde{\delta}(V, W)$.

On peut alors conjecturer :

Conjecture 1.3. Soient $V$ une sous-variété de $\mathbb{G}_{m}^{n}$, et $B$ le plus petit translaté d'un sous-tore contenant $V$. Alors, l'ensemble des points $\boldsymbol{x}$ de $V^{\circ}(\overline{\mathbb{Q}})$ de hauteur

$$
\hat{h}(\boldsymbol{x}) \leqslant \frac{c(n)^{-1} \operatorname{deg}(B)}{\tilde{\delta}(V, B)}
$$

est de cardinal fini, majoré par

$$
\frac{c(n) \tilde{\delta}(V, B)^{b}}{\operatorname{deg}(B)^{b-1}}
$$

où $b$ est la dimension de $B$ et $c(n)>1$.

Remarques. En particulier, cette conjecture affirme que

$$
\hat{\mu}^{\circ}(V) \geqslant \frac{c(n)^{-1} \operatorname{deg}(B)}{\tilde{\delta}(V, B)} .
$$




\section{Points DE PETITE hauteur}

Il est intéressant de noter qu'en tout état de cause, $\tilde{\delta}(V)$ est majoré par $\operatorname{deg}(V)$. De plus, on voit facilement que $\tilde{\delta}(V, B) \leqslant \operatorname{deg}(B) \tilde{\delta}(V)$. Ainsi, la conjecture 1.3 entraîne en particulier :

$$
\hat{\mu}^{\circ}(V) \geqslant \frac{c(n)^{-1}}{\operatorname{deg}(V)} \text {. }
$$

Enfin, il ne semble pas possible de conjecturer une minoration dépendant d'un invariant comme l'indice d'obstruction, comme le montre l'exemple ci-dessous. La conjecture 1.3 semble donc être essentiellement optimale quant au comportement de $\hat{\mu}^{\circ}(V)$ en fonction du degré de $V$.

Exemple. Soit $m$ un entier $\geqslant 1$; considérons la sous-variété $V$ de $\mathbb{G}_{m}^{4}$ définie par les équations :

$$
F(x, y, z, t):=x^{m}+y^{m}-1=0, \quad G(x, y, z, t):=x^{2}+x^{3}-z-t=0 .
$$

On vérifie facilement que $V^{\circ}=V$, et que $V$ n'est contenue dans aucun translaté de sous-tore strict de $\mathbb{G}_{m}^{n}$. Enfin, on voit aussi que $\omega(V) \leqslant 3$ (en fait $\omega(V)=3$ ). Soit $\mathcal{C}$ la courbe $\subset \mathbb{G}_{m}^{2}$ définie par $F=0$ (vu comme polynôme de deux variables) et soit enfin $\left(\alpha_{n}, \beta_{n}\right)$ une suite de points de $\mathcal{C}(\overline{\mathbb{Q}})$ telle que $\hat{h}\left(\alpha_{n}, \beta_{n}\right)$ converge vers $\hat{\mu}^{\text {ess }}(\mathcal{C}) \sim 1 / m$. On pose

$$
P_{n}:=\left(\alpha_{n}, \beta_{n}, \alpha_{n}^{2}, \alpha_{n}^{3}\right) .
$$

Les points $P_{n}$ sont donc tous dans $V^{\circ}$, leur hauteur vérifie $\hat{h}\left(P_{n}\right) \leqslant 3 \hat{h}\left(\alpha_{n}, \beta_{n}\right)$. Ainsi, on a exhibé une suite de points de $V^{\circ}$ de hauteur $\leqslant c / m$ ce qui montre bien que $\hat{\mu}^{\circ}(V) \leqslant c / m$. Pour $m$ assez grand, ceci contredirait l'inégalité $\hat{\mu}^{\circ}(V) \geqslant c / \omega(V)$.

Toutefois, il n'est pas exclu que l'on puisse se contenter d'un indice de quasi interpolation défini comme suit.

Définition 1.4. Si $V \subsetneq W$, l'indice de quasi interpolation de $V$ relativement à $W$ est le plus petit entier $\delta$ pour lequel il existe des sous-variétés $Y_{1}, \ldots, Y_{t}$ de $W$, de degré $\leqslant \delta$ telles que $V$ soit une composante isolée de $Y_{1} \cap \cdots \cap Y_{t}$. On le notera $\delta(V, W)$, et simplement $\delta(V)$ si $W=\mathbb{P}^{n}$.

Bien entendu, on dispose des inégalités :

$$
\omega(V) \leqslant \delta(V) \leqslant \tilde{\delta}(V) \leqslant \operatorname{deg}(V) .
$$

De plus, $\delta(V)=\tilde{\delta}(V)$ si $V$ est une intersection complète.

Dans la direction de la conjecture 1.3, nous obtenons le théorème suivant :

ThÉORÈme 1.5. Soit $V$ une sous-variété de $\mathbb{G}_{m}^{n}$. On a :

$$
\hat{\mu}^{\circ}(V)>c^{\prime}(n)^{-1} \delta(V)^{-1}(\log (3 \delta(V)))^{-\lambda(n-1)},
$$

où $c^{\prime}(n)>1$ et $\lambda(k)=\left(9(3 k)^{(k+1)}\right)^{k}$ est la fonction de $k$ introduite au théorème 1.1. Il existe donc un nombre fini de translatés de sous-tores $B_{1}, \ldots, B_{m}$ contenus dans $V$ tels que

$$
\hat{h}(\boldsymbol{\alpha}) \geqslant c^{\prime}(n)^{-1} \delta(V)^{-1}(\log (3 \delta(V)))^{-\lambda(n-1)},
$$

pour tout

$$
\boldsymbol{\alpha} \in V(\overline{\mathbb{Q}}), \quad \boldsymbol{\alpha} \notin \bigcup_{j=1}^{m} B_{j}
$$

\section{Remarques.}

(i) Le théorème 1.5 établit à une puissance du logarithme du degré près la minoration conjecturée pour le dernier des minima géométriques, tout au moins lorsque le degré du groupe ambiant est négligé (comparer avec la conjecture 1.3 avec $B=\mathbb{G}_{m}^{n}$ ). 


\section{F. Amoroso et S. David}

(ii) Ce résultat semble indiquer qu'au moins pour les compactifications naturelles du groupe multiplicatif $\mathbb{G}_{m}^{n}$, on puisse conjecturer que le bon invariant pour ce problème est bien $\delta(V)$.

(iii) Rappelons que la meilleure minoration connue précédemment était due au second auteur et à Philippon (confer [DP99, théorème 1.3]) et affirmait que si

$$
q(V):=\left(2^{n+4 \operatorname{dim}(V)+22} \operatorname{deg}(V)(\log (\operatorname{deg}(V)+1))^{2 / 3}\right)^{7^{\operatorname{dim}(V)},},
$$

alors, l'ensemble des points de $V^{\circ}(\overline{\mathbb{Q}})$ de hauteur

$$
\hat{h}(\boldsymbol{x}) \leqslant \frac{1}{q(V)^{3 / 4}}
$$

est fini, de cardinal au plus $q(V)$, soit une minoration de $\hat{\mu}^{\circ}(V)$ essentiellement du type $\operatorname{deg}(V)^{-7^{\operatorname{dim}(V)}}$, à comparer à $\delta(V)^{-1-\varepsilon}$ dans le théorème 1.5. Les résultats antérieurs (confer Bombieri et Zannier [BZ95] et Schmidt [Sch96]) étaient quant à eux pluri-exponentiels en le degré de $V$.

Si la minoration de $\hat{\mu}^{\circ}(V)$ dans le théorème 1.5 est assez satisfaisante (à l'exception peut-être des valeurs de $c(n)$ et $\lambda(n-1)$ ), nous ne disons rien ici sur le nombre de points exceptionnels. Rappelons qu'une des applications d'énoncés comme le théorème 1.5 est une estimation pour le nombre de points de hauteur bornée de $V^{\circ}$ se trouvant dans un sous-groupe de rang fini. Plus précisément, soient $\Gamma \subset \mathbb{G}_{m}^{n}(\overline{\mathbb{Q}})$ un sous-groupe de rang $\leqslant r$, et $a$ un nombre réel positif. On cherche à majorer le nombre $N$ de points de $V^{\circ}(\overline{\mathbb{Q}}) \cap \Gamma$ de hauteur $\leqslant a$. Ainsi, du théorème 1.3 de [DP99], on déduit que (confer [DP99, théorème 1.5])

$$
N \leqslant a^{r} \cdot q(V)^{r+1} .
$$

Une telle borne est pour sa part cruciale pour les applications ultérieures. On pourra se reporter en particulier aux travaux de Evertse et Schlickewei (confer [ES02]) et de Rémond (confer [Rém02]) pour plus de détails sur ces questions. Pour parvenir à une telle estimation, on ne peut se contenter d'une minoration (même très bonne) pour $\hat{\mu}^{\circ}(V)$ : il faut parallèlement conserver un contrôle sur le nombre de points exceptionnels (i.e. de hauteur exceptionnellement petite) . Toutefois, si les méthodes introduites ici sont très performantes pour la hauteur, elles ne le sont guère pour le nombre de points exceptionnels, et les résultats seraient très comparables à ceux déjà obtenus dans [DP99].

On peut plus généralement chercher à estimer la quantité

$$
D:=\sum_{j=1}^{m} \operatorname{deg} B_{j}
$$

(voir la formulation du théorème 1.5$)^{5}$ en fonction du paramètre $\tilde{\delta}(V)$. Bien sûr, l'entier $D$ majore le nombre de points exceptionnels dans $V^{\circ}$, mais on peut dire plus : on contrôle ainsi la distribution des points exceptionnels dans l'ensemble de $V$ (et donc en particulier sur le fermé $V \backslash V^{\circ}$ ). Par exemple, on obtient ainsi des informations précises même si $V^{\circ}$ est vide.

On peut noter qu'un énoncé de ce type est en fait démontré dans [DP99] (confer la proposition 5.6) : l'ensemble des points de $V(\overline{\mathbb{Q}})$ de hauteur $\leqslant q(V)^{-3 / 4}$ est contenu dans une réunion finie de translatés de sous-tores $B_{1}^{\prime}, \ldots, B_{m^{\prime}}^{\prime}$ contenus dans $V$ tels que $\sum_{j=1}^{m^{\prime}} \operatorname{deg}\left(B_{j}^{\prime}\right) \leqslant q(V)$.

Il semble raisonnable de renforcer la conjecture 1.3 pour inclure dans les exceptions les translatés par des sous-tores non triviaux, et conjecturer (en reprenant les notations de la conjecture 1.3) que

${ }^{5} \mathrm{Il}$ convient de remarquer que $D$ est en fait fonction de la minoration demandée pour la hauteur. 


\section{Points DE PETITE hauteur}

l'ensemble des points de $V(\overline{\mathbb{Q}})$ de hauteur

$$
\leqslant \frac{c(n)^{-1} \operatorname{deg}(B)}{\tilde{\delta}(V, B)}
$$

est contenu dans une réunion de translatés de sous-tores contenus dans $V$ dont la somme $D$ des degrés est majorée par :

$$
D \leqslant \frac{c(n) \tilde{\delta}(V, B)^{b}}{\operatorname{deg}(B)^{b-1}} .
$$

De plus, dans le cadre du théorème $1.5\left(B=\mathbb{G}_{m}^{n}\right)$, une borne du type

$$
D \leqslant c^{\prime}(n) \delta(V)^{n}(\log (3 \delta(V)))^{\kappa(n)}
$$

pour la somme $D:=\sum_{j=1}^{m} \operatorname{deg} B_{j}$, est peut-être accessible à l'aide des techniques actuelles. ${ }^{6}$ En dehors de son intérêt propre, un tel résultat aurait notamment comme conséquence la borne suivante pour le nombre $N$ de points de $V^{\circ}(\overline{\mathbb{Q}}) \cap \Gamma$ de hauteur $\leqslant a$ :

$$
N \leqslant c(n, \varepsilon) a^{r} \delta(V)^{r(n+1)+\varepsilon},
$$

une amélioration substantielle du théorème 1.4 de [DP99].

Il est intéressant de noter qu'en fait une borne polynomiale en le degré de $V$ pourrait être obtenue pour $D$ (essentiellement de l'ordre $\operatorname{de} \operatorname{deg}(V)^{7^{\operatorname{dim}(V)}}$ ), en combinant les méthodes du paragraphe 5 de [DP99] (confer en particulier la proposition 5.6 de ce texte) avec celles du présent article.

Signalons enfin que des résultats partiels vers ce type de questions pour des surfaces dans $\mathbb{G}_{m}^{3}$ ont été récemment obtenus par Pontreau (confer [Pon05]).

La preuve du théorème 1.5 occupera le paragraphe 3 . Ce dernier s'obtient à partir d'une variante quantitative du théorème 1.1 (le théorème 2.1), à l'aide d'une récurrence sur la dimension qui est assez proche de l'argument que l'on trouvera dans notre texte [AD03b] qui traitait pour sa part du « dernier minimum arithmétique ». Il est à noter que la récurrence est très différente de celle employée dans [DP99] (arguments d'intersection, et théorèmes de Bézout) qui est elle très coûteuse en terme de qualité des bornes. La preuve du théorème 2.1 occupe pour sa part le paragraphe 2 . Comme cette dernière est très proche de celle du théorème 1.1, nous avons, pour garder à ce texte une longueur raisonnable et éviter trop de redites, fait appel très souvent aux arguments de [AD03a]. Il est donc conseillé au lecteur de garder cette référence à sa portée pour suivre les démonstrations. Que le lecteur préférant un texte plus auto-explicite veuille bien nous en excuser.

C'est un plaisir pour nous de remercier ici chaleureusement l'arbitre qui par ses remarques a contribué à préciser des points par rapport à notre manuscrit initial et améliorer la présentation générale de ce texte.

\section{Résultats auxiliaires}

Nous précisons dans ce paragraphe le théorème 1.1 : dans [AD03a], nous avons supposé que $V$ n'est contenue dans aucun translaté d'un sous-tore propre de $\mathbb{G}_{m}^{n}$, pour minorer son minimum essentiel en fonction de $\omega(V)$. Toutefois, comme c'est souvent le cas en géométrie diophantienne on peut, dans le cas dégénéré, obtenir une information quantitative précise. Ici, cela reviendrait à proposer l'alternative suivante : ou bien le minimum essentiel de $V$ est assez grand, ou bien $V$ est contenue dans un translaté $B$ d'un sous-tore strict de $\mathbb{G}_{m}^{n}$ dont le degré est contrôlé précisément en fonction des données du problème (ici, $\omega(V)$, la dimension du groupe ambiant et la dimension de $V$ ). C'est très précisément ce qui se produit :

${ }^{6}$ Quitte à modifier éventuellement les fonctions $\lambda(k)$ et $c^{\prime}(n)$. 


\section{F. Amoroso et S. David}

ThÉorème 2.1. Pour tout entier $n \geqslant 1$, il existe un nombre réel strictement positif $c^{\prime}(n)$, effectivement calculable, tel que la propriété suivante soit vraie. Soit $V$ une sous-variété de $\mathbb{G}_{m}^{n}$ de codimension $k$. Alors, ou bien il existe un translaté d'un sous-tore $B \subsetneq \mathbb{G}_{m}^{n}$ contenant $V$, tel que :

$$
(\operatorname{deg}(B))^{1 / \operatorname{codim}(B)} \leqslant c^{\prime}(n) \omega(V)(\log (3 \omega(V)))^{\lambda(k)},
$$

ou bien :

$$
\hat{\mu}^{\mathrm{ess}}(V) \geqslant c^{\prime}(n)^{-1} \omega(V)^{-1}(\log (3 \omega(V)))^{-\lambda(k)} ;
$$

comme dans le théorème 1.1, le nombre $\lambda(k)$ est explicitement connu et vaut :

$$
\lambda(k):=\left(9(3 k)^{(k+1)}\right)^{k} .
$$

Démonstration. On suit la preuve du théorème 1.4 de [AD03a] dont nous conservons les notations ; nous indiquons simplement les passages où il convient d'être plus précis.

Soit $V$ une sous-variété propre et $\overline{\mathbb{Q}}$-irréductible de $\mathbb{G}_{m}^{n}$ de codimension $k$ dont on suppose qu'elle a un petit minimum essentiel :

$$
\hat{\mu}^{\text {ess }}(V)<c^{\prime}(n)^{-1} \omega(V)^{-1}(\log (3 \omega(V)))^{-\lambda(k)} ;
$$

Nous pouvons alors appliquer la proposition 5.5 de $[\mathrm{AD} 03 \mathrm{a}]^{7}$ : en particulier, on est assuré de l'existence de deux sous-variétés propres et $\overline{\mathbb{Q}}$-irréductibles $W_{i-1}$ et $W_{i}$ de $\mathbb{G}_{m}^{n}$, de la même dimension, et de l'existence de certains entiers $l_{1}, \ldots, l_{i} \in \mathbb{N}^{*}$ qui vérifient :

$$
\operatorname{dim}\left(W_{i-1}\right)=\operatorname{dim}\left(W_{i}\right), \quad\left[l_{i}\right] W_{i-1} \subseteq W_{i}, \quad\left[l_{1} \ldots l_{i-1}\right] V \subseteq W_{i} .
$$

De plus, $l_{i}$ est premier avec $\left[\operatorname{Stab}\left(W_{i-1}\right): \operatorname{Stab}\left(W_{i-1}\right)^{0}\right]$. Si $W_{i}$ n'est pas un translaté d'un sous-tore propre de $\mathbb{G}_{m}^{n}$, alors la suite de la preuve du théorème 1.4 de [AD03a] conduit à une contradiction. On peut donc supposer que $W_{i}$ est un translaté d'un sous-tore propre ; il s'ensuit que

$$
\tilde{B}:=\left[l_{1} \ldots l_{i-1}\right]^{-1} W_{i}
$$

est une réunion de translatés d'un sous-tore propre et contient $V\left(\right.$ car $W_{i}$ contient $\left.\left[l_{1} \ldots l_{i-1}\right] V\right)$. Choisissons une composante, que nous noterons $B$, de $\tilde{B}$ contenant $V$. Pour démontrer le théorème 2.1, il suffit de majorer le degré de $B$.

En appliquant les relations (ii) de la définition 5.3 et la scolie 5.4 de [AD03a], on tire :

$$
\left(\operatorname{deg}\left(W_{i}\right)\right)^{1 / \operatorname{codim}\left(W_{i}\right)} \leqslant\left(P_{i+1} \ldots P_{k}\right)^{2} \omega\left(\left[l_{1} \ldots l_{i}\right] V\right) \leqslant\left(P_{1} \ldots P_{k}\right)\left(P_{i+1} \ldots P_{k}\right) \omega(V),
$$

d'où (rappelons que $W_{i}$ est un translaté d'un sous-tore propre de $\mathbb{G}_{m}^{n}$ )

$$
(\operatorname{deg}(B))^{1 / \operatorname{codim}(B)}=\left(\operatorname{deg}\left(W_{i}\right)\right)^{1 / \operatorname{codim}\left(W_{i}\right)} \leqslant\left(P_{1} \ldots P_{k}\right)^{2} \omega(V) .
$$

Par ailleurs, $\left(P_{1} \ldots P_{k}\right)^{2}=\left(C_{0}^{2} \log (3 \omega(V))\right)^{u}$, où (confer les choix des paramètres $P_{i}$ et l'inégalité (5.2) dans $[\mathrm{AD} 03 \mathrm{a}])$ :

$$
u:=4(3 k)^{k+1}\left(\rho_{1}+\cdots+\rho_{k}\right) \leqslant 8(3 k)^{k+1} \rho_{1}<\left(9(3 k)^{(k+1)}\right)^{k}=\lambda(k) .
$$

On a donc :

$$
(\operatorname{deg}(B))^{1 / \operatorname{codim}(B)} \leqslant \omega(V)\left(C_{0}^{2} \log (3 \omega(V))\right)^{\lambda(k)} .
$$

Le théorème 2.1 est donc entièrement établi.

On se propose, au paragraphe 3 , de déduire le théorème 1.5 du résultat précédent, au moyen d'une récurrence sur la dimension du groupe ambiant, analogue à celle utilisée dans la preuve du

\footnotetext{
${ }^{7}$ L'hypothèse que $V$ n'est contenue dans aucun translaté de sous-tore propre de $\mathbb{G}_{m}^{n}$, qui apparaît dans l'énoncé de cette proposition, n'est en fait pas utilisée dans sa preuve. Cette dernière n'apparaît qu'à une étape ultérieure de la démonstration, au paragraphe 5.3.
} 


\section{Points De Petite hauteur}

théorème 1.4 de [AD03b]. Pour ce faire, nous avons d'abord besoin d'exprimer $\hat{\mu}^{\circ}(V)$ en fonction du minimum essentiel de certaines sous-variétés de $V$ :

Lemme 2.2. Soit $V$ une sous-variété de $\mathbb{G}_{m}^{n}$. On a alors :

$$
\hat{\mu}^{\circ}(V)=\inf _{Z}\left\{\hat{\mu}^{\text {ess }}(Z)\right\},
$$

où l'infimum est pris sur les sous-variétés $Z$ de $V$, qui ne sont pas contenues dans un translaté $B \subseteq V$ d'un sous-tore de $\mathbb{G}_{m}^{n}$.

Démonstration. Nous commençons par montrer que $\inf _{Z}\left\{\hat{\mu}^{\text {ess }}(Z)\right\} \geqslant \hat{\mu}^{\circ}(V)$; soit donc $\theta$ un nombre réel strictement supérieur à $\inf _{Z}\left\{\hat{\mu}^{\text {ess }}(Z)\right\}$. Par définition, il existe donc une sous-variété $Z$ de $V$, qui n'est pas contenue dans un translaté $B$ d'un sous-tore de $\mathbb{G}_{m}^{n}$ tel que $B \subseteq V$ et telle que $Z(\theta)$ est Zariski-dense dans $Z$. Supposons que $V(\theta)$ soit contenu dans une réunion finie $\Sigma$ de translatés de sous-tores contenus dans $V$. On a alors : $Z(\theta) \subseteq \Sigma$ et donc $Z \subseteq \Sigma$ par densité. On en déduit que $Z$ est contenu dans une composante irréductible de $\Sigma$, i.e. dans un translaté $B \subseteq V$ d'un sous-tore (car $Z$ est irréductible) ; c'est une contradiction. En conclusion, $V(\theta)$ n'est pas contenu dans une réunion finie de translatés de sous-tores contenus dans $V$, d'où, par définition de $\hat{\mu}^{\circ}(V)$,

$$
\theta \geqslant \hat{\mu}^{\circ}(V) \text {. }
$$

Montrons maintenant que $\inf _{Z}\left\{\hat{\mu}^{\text {ess }}(Z)\right\} \leqslant \hat{\mu}^{\circ}(V)$. Pour cela, soit $\theta$ un réel, $\theta>\hat{\mu}^{\circ}(V)$; l'ensemble $V(\theta)$ n'est donc pas contenu dans une réunion finie de translatés de sous-tores contenus dans $V$. On en déduit l'existence d'une composante irréductible $Z$ de la clôture de Zariski de $V(\theta)$ qui n'est pas contenue dans un translaté $B \subseteq V$ d'un sous-tore de $\mathbb{G}_{m}^{n}$. Remarquons que $V(\theta) \cap Z$ est Zariski-dense dans $Z$. Donc $\hat{\mu}^{\text {ess }}(Z) \leqslant \theta$. Ce qui donne :

$$
\inf _{Z}\left\{\hat{\mu}^{\text {ess }}(Z)\right\} \leqslant \theta \text {. }
$$

Le lemme 2.2 est donc établi.

\section{Démonstration du théorème 1.5}

Nous pouvons maintenant démontrer le théorème 1.5. Nous allons procéder par récurrence sur $n$. Dans le principe, nous suivons la démarche introduite ${ }^{8}$ pour la preuve du théorème 1.4 de [AD03b] : si une sous-variété $Z$ de $V$ a un trop petit minimum essentiel, alors, elle est contenue dans un translaté d'un sous-groupe, dont nous contrôlons le degré par le théorème 2.1. On peut alors utiliser une bonne paramétrisation de ce sous-groupe pour se ramener à une situation en dimension ambiante plus petite.

Grâce au lemme 2.2, il suffit de montrer que pour tout $n \in \mathbb{N}$, pour toute sous-variété $V$ de $\mathbb{G}_{m}^{n}$, et pour toute sous-variété $Z$ de $V$ qui n'est pas contenue dans un translaté $B \subseteq V$ d'un sous-tore, on a :

$$
\hat{\mu}^{\mathrm{ess}}(Z)>c^{\prime}(n)^{-1} \delta(V)^{-1}(\log (3 \delta(V)))^{-\lambda(n-1)},
$$

pour un certain nombre réel $c^{\prime}(n)>0$.

\footnotetext{
${ }^{8}$ Dans [AD03b], nous nous intéressons au minimum pour la hauteur des points dans l'ouvert $V^{\star}$ de $V$, c'est-à-dire $V$ privé de la réunion des sous-variétés de torsion contenues dans $V$. Pour ce minimum, on ne peut espérer minorer la hauteur uniquement en fonction de données issues de la géométrie de $V$; le degré d'un corps de définition pour $V$ intervient nécessairement : en dimension $n=1, V$ est la réunion des conjugués d'un nombre algébrique (non de torsion), $V^{\star}=V$ et l'on est ramené au problème de Lehmer classique. En contrepartie, on peut obtenir une minoration valable pour tous les points sans exception de $V^{\star}$. De même, les méthodes de preuve pour les deux questions (minimum sur $V^{\circ}$ ou $V^{\star}$ ) diffèrent. Toutefois, de nombreuses similitudes existent entre les deux questions, et il n'est donc pas surprenant que certains arguments soient communs ou tout au moins voisins.
} 


\section{F. Amoroso et S. David}

Si $n=1$, cette propriété est vraie ( $V$ est un point, donc un translaté d'un sous-tore contenu dans celui-ci). Supposons donc cette propriété vraie pour un certain entier $n-1 \geqslant 1$ et supposons par l'absurde qu'elle soit fausse pour $n$. Soit $C_{0}(n)$ un nombre réel assez grand (que l'on pourrait calculer effectivement à partir de la constante $c(n)$ du théorème 2.1). Il existe alors un entier $\delta \geqslant 1$, une sous-variété algébrique $\overline{\mathbb{Q}}$-irréductible $V$ de $\mathbb{G}_{m}^{n}$ telle que $\delta(V) \leqslant \delta$ et une sous-variété $Z \subseteq V$ de codimension $k$, qui n'est pas contenue dans un translaté $B \subseteq V$ d'un sous-tore et telle que

$$
\hat{\mu}^{\text {ess }}(Z)<C_{0}(n)^{-1} \delta^{-1}(\log (3 \delta))^{-\lambda(n-1)} .
$$

Remarquons que $\omega(Z) \leqslant \delta$ (puisque $Z \subseteq V$, par définition $\omega(Z) \leqslant \omega(V) \leqslant \delta(V)$ ) et que $\lambda(k) \leqslant \lambda(n-1)$. Donc

$$
\hat{\mu}^{\mathrm{ess}}(Z)<C_{0}(n)^{-1} \omega(Z)^{-1}(\log (3 \omega(Z)))^{-\lambda(k)}
$$

et le théorème 2.1 nous assure que $Z$ est contenue dans un certain translaté $\boldsymbol{\alpha} \cdot H$ d'un sous-tore $H \subsetneq \mathbb{G}_{m}^{n}$ de codimension $k^{\prime} \leqslant k$, dont le degré vérifie l'inégalité :

$$
(\operatorname{deg}(H))^{1 / k^{\prime}} \leqslant c(n) \delta(\log (3 \delta))^{\lambda(n-1)} .
$$

De plus, il n'y a pas de restriction à supposer que $\boldsymbol{\alpha} \in Z$ et est tel que $\hat{h}(\boldsymbol{\alpha}) \leqslant 2 \hat{\mu}^{\text {ess }}(Z)$; on a alors :

$$
\hat{\mu}^{\text {ess }}\left(\boldsymbol{\alpha}^{-1} \cdot Z\right) \leqslant \hat{h}\left(\boldsymbol{\alpha}^{-1}\right)+\hat{\mu}^{\text {ess }}(Z) \leqslant n \hat{h}(\boldsymbol{\alpha})+\hat{\mu}^{\text {ess }}(Z) \leqslant 3 n \hat{\mu}^{\text {ess }}(Z) .
$$

Fixons une base du sous-module

$$
\Lambda:=\left\{\boldsymbol{\lambda} \in \mathbb{Z}^{n}, \text { tel que } \forall \boldsymbol{x} \in H, \boldsymbol{x}^{\boldsymbol{\lambda}}=1\right\} \subseteq \mathbb{Z}^{n}
$$

des relations de $H$ et notons $A$ la matrice $n \times k^{\prime}$ dont les lignes sont formées par les coordonnées de cette base par rapport à la base canonique de $\mathbb{R}^{n}$. Notons $E=\Lambda \otimes_{\mathbb{Z}} \mathbb{R}$. On sait alors (confer [BP88]) que le degré de $H$ est égal au maximum des valeurs absolues des déterminants des mineurs $k^{\prime} \times k^{\prime}$ de $A$ et que le volume $\operatorname{Vol}(E / \Lambda)$ de $\Lambda$ (par rapport à la métrique euclidienne usuelle) est égal à la moyenne quadratique des mêmes déterminants. On a alors :

$$
\operatorname{Vol}(E / \Lambda) \leqslant\left(\begin{array}{l}
n \\
k^{\prime}
\end{array}\right)^{1 / 2} \operatorname{deg}(H) \leqslant n^{k^{\prime}} \operatorname{deg}(H) .
$$

Soit $C$ le cube $[-1 / 2,1 / 2]^{n} \subset \mathbb{R}^{n}$; par un théorème de Vaaler (confer [Vaa79]), on a

$$
\operatorname{Vol}(C \cap E) \geqslant 1
$$

et donc le théorème de Minkowski nous fournit un élément non nul $\boldsymbol{\lambda} \in \Lambda$ tel que :

$$
D:=\max _{1 \leqslant i \leqslant n}\left\{\left|\lambda_{i}\right|\right\} \leqslant n \operatorname{deg}(H)^{1 / k^{\prime}} .
$$

On peut supposer que $\lambda_{1}, \ldots, \lambda_{n}$ sont globalement premiers entre eux (car $H$ est connexe) ; quitte à renuméroter les variables et à changer les signes, on peut également supposer $\lambda_{n}=D$.

Le sous-tore $H^{\prime}$ d'équation

$$
\boldsymbol{x}^{\boldsymbol{\lambda}}=1
$$

contient donc $H$, il est de degré au plus $n D$ et on dispose de la paramétrisation $\varphi: \mathbb{G}_{m}^{n-1} \rightarrow H^{\prime}$ définie par :

$$
\varphi(\boldsymbol{x}):=\left(x_{1}^{\lambda_{n}}, \ldots, x_{n-1}^{\lambda_{n}}, x_{1}^{-\lambda_{1}}, \ldots, x_{n-1}^{-\lambda_{n-1}}\right) .
$$

Soit $Z^{\prime}$ une composante irréductible de $\varphi^{-1}\left(\boldsymbol{\alpha}^{-1} \cdot Z\right)$ et notons $V^{\prime}$ une composante irréductible de $\varphi^{-1}\left(\boldsymbol{\alpha}^{-1} \cdot V\right)$ contenant $Z^{\prime}$. On a alors le suivant. 


\section{Points DE PETITE hauteur}

(a) La sous-variété $V^{\prime}$ de $\mathbb{G}_{m}^{n-1}$ est une composante irréductible du lieu des zéros de polynômes dont le degré satisfait l'inégalité :

$$
\leqslant \max \left\{\lambda_{n},\left|\lambda_{1}+\cdots+\lambda_{n-1}\right|\right\} \delta \leqslant n D \delta ;
$$

en particulier, $\delta\left(V^{\prime}\right) \leqslant n D \delta$.

(b) Par construction, $Z^{\prime} \subseteq V^{\prime}$ et $Z^{\prime}$ n'est contenue dans aucun translaté d'un sous-tore inclus dans $V^{\prime}$. En effet, si $Z^{\prime} \subseteq B^{\prime}$ pour un certain translaté d'un sous-tore $B^{\prime} \subseteq V^{\prime}$, alors on aurait ${ }^{9}$ $Z=\boldsymbol{\alpha} \cdot \varphi\left(Z^{\prime}\right) \subseteq B$ avec $B:=\boldsymbol{\alpha} \cdot \varphi\left(B^{\prime}\right) \subseteq V$ translaté d'un sous-tore, ce qui contredit le choix de $Z$.

(c) Les minima essentiels de $Z$ et $Z^{\prime}$ sont liés par l'inégalité (confer la relation (3)) :

$$
3 n \hat{\mu}^{\text {ess }}(Z) \geqslant \hat{\mu}^{\text {ess }}\left(\boldsymbol{\alpha}^{-1} \cdot Z\right)=\hat{\mu}^{\text {ess }}\left(\varphi\left(Z^{\prime}\right)\right) \geqslant \lambda_{n} \hat{\mu}^{\text {ess }}\left(Z^{\prime}\right)=D \hat{\mu}^{\text {ess }}\left(Z^{\prime}\right) \text {. }
$$

Par hypothèse de récurrence, il existe une constante $c^{\prime}(n-1)>0$ telle que

$$
3 n D^{-1} \hat{\mu}^{\text {ess }}(Z) \geqslant \hat{\mu}^{\text {ess }}\left(Z^{\prime}\right)>c^{\prime}(n-1)^{-1}(n D \delta)^{-1}(\log (3 n D \delta))^{-\lambda(n-2)},
$$

d'où, compte tenu de la majoration (4),

$$
\hat{\mu}^{\text {ess }}(Z) \geqslant c^{\prime}(n-1)^{-1}\left(3 n^{2}\right)^{-1} \delta^{-1}\left(\log \left(3 n^{2} \operatorname{deg}(H) \delta\right)\right)^{-\lambda(n-2)} .
$$

L'inégalité (2) majorant le degré de $H$, on voit pour finir que l'on peut choisir la constante $C_{0}(n)$ assez grande de telle sorte que :

$$
3 n^{2} c^{\prime}(n-1)\left(\log \left(3 n^{2} \operatorname{deg}(H) \delta\right)\right)^{\lambda(n-2)} \leqslant C_{0}(n)(\log (3 \delta))^{\lambda(n-2)} .
$$

Pour un tel choix de $C_{0}(n)$ on a nécessairement :

$$
\begin{aligned}
\hat{\mu}^{\text {ess }}(Z) & \geqslant C_{0}(n)^{-1} \delta^{-1}(\log 3 \delta)^{-\lambda(n-2)} \\
& \geqslant C_{0}(n)^{-1} \delta^{-1}(\log 3 \delta)^{-\lambda(n-1)} .
\end{aligned}
$$

On aboutit à une contradiction, ce qui termine la preuve du théorème 1.5.

\section{BIBLIOGRAPHIE}

Amo04 F. Amoroso, Small points on subvarieties of algebraic tori: results and methods, Riv. Mat. Univ. Parma (7) 3 (2004), 1-31.

AD03a F. Amoroso et S. David, Minoration de la hauteur normalisée dans un tore, J. Inst. Math. Jussieu 2 (2003), 335-381.

AD03b F. Amoroso et S. David, Distribution des points de petite hauteur dans les groupes multiplicatifs, Ann. Scuola Norm. Sup. Pisa Cl. Sci. (4) 3 (2003), 325-348.

BP88 D. Bertrand et P. Philippon, Sous-groupes algébriques de groupes algébriques commutatifs, Illinois J. Math. 32 (1988), 263-280.

BZ95 E. Bombieri et U. Zannier, Algebraic points on subvarieties of $\mathbb{G}_{m}^{n}$, Int. Math. Res. Not. 7 (1995), 333-347.

Cha88 M. Chardin, Une majoration de la fonction de Hilbert et ses conséquences pour l'interpolation algébrique, Bull. Soc. Math. France 117 (1988), 305-318.

Voir aussi Contributions à l'algèbre commutative effective et à la théorie de l'élimination, Thèse de doctorat, Université de Paris VI (1990).

Dav S. David, On the height of subvarieties of groups varieties, Ramanujan J., à paraître.

DP99 S. David et P. Philippon, Minorations des hauteurs normalisées des sous-variétés des tores, Ann. Scuola Norm. Sup. Pisa Cl. Sci. (4) 28 (1999), 489-543. Errata 29 (2000), 729-731.

\footnotetext{
${ }^{9}$ Rappelons que comme $Z \subseteq \boldsymbol{\alpha} \cdot H$, on a nécessairement $\boldsymbol{\alpha} \cdot \varphi\left(Z^{\prime}\right)=Z$.
} 


\section{Points DE PETite HaUteur}

ES02 J.-H. Evertse et H.-P. Schlickewei, A quantitative version of the Absolute Subspace Theorem, J. reine angew. Math. 548 (2002), 21-127.

Pon05 C. Pontreau, Small points on surfaces, Preprint (2005).

Rém02 G. Rémond, Sur les sous-variétés des tores, Compositio Math. 134 (2002), 337-366.

Sch96 W. Schmidt, Heights of points on subvarieties of $\mathbb{G}_{m}^{n}$, in Number theory, Séminaire de théorie des nombres de Paris 1993-1994, London Mathematical Society Lecture Note Series, vol. 235, éd. S. David (Cambridge University Press, Cambridge, 1996), 157-187.

Vaa79 J. D. Vaaler, A geometric inequality with applications to linear forms, Pacific J. Math. 83 (1979), 543-553.

Zha92 S. Zhang, Positive line bundles on arithmetic surfaces, Ann. of Math. (2) 136 (1992), 569-587.

Zha95 S. Zhang, Positive line bundles on arithmetic varieties, J. Amer. Math. Soc. 8 (1995), 187-221.

Francesco Amoroso amoroso@math.unicaen.fr

UMR 6139 (CNRS), Nicolas Oresme, Département de Mathématiques, Université de Caen, Campus II, BP 5186, F-14032 Caen cedex, France

Sinnou David david@math.jussieu.fr

UMR 7586 (CNRS) - UFR 921, Théorie des nombres, Institut de Mathématiques de Jussieu, Université Pierre et Marie Curie, 4, Place Jussieu, 75005 Paris, France 